\title{
Modeling of Ultrasonic Guided-Wave Reflection from a Discontinuity in a Plate Structure*
}

\author{
Yoshiaki NAGASHIMA**, Masao ENDOU**, Masahiro MIKI**, \\ Yoshio NONAKA*** and Ken'ichi ASAMI**** \\ **Hitachi Research Laboratory, Hitachi, Ltd., \\ 7-2-1 Omika-cho, Hitachi-shi, Ibaraki-ken, 319-1221, Japan \\ E-mail: yoshiaki.nagashima.ka@hitachi.com \\ ***Hitachi-GE Nuclear Energy, Ltd., \\ 3-1-1 Saiwai-cho, Hitachi-shi, Ibaraki-ken, 317-0073, Japan \\ ****Hitachi Power Solutions Co., Ltd., \\ 3-2-2 Saiwai-cho, Hitachi-shi, Ibaraki-ken, 317-0073, Japan
}

\begin{abstract}
In this paper, a combined model was proposed for reflection from a discontinuity and for a wave field of a fundamental shear horizontal guided wave in a plate structure. The reflection coefficient of a guided wave from a rectangular discontinuity can be modeled in the same way as the total reflection coefficient of a bulk wave. For the discontinuity having a slightly changed cross-sectional area, the reflection coefficient from the whole discontinuity can be calculated by spatial integration of the waves reflected from the divided regions. Some experiments and analyses were performed for $4 \mathrm{~mm}$-thick stainless steel plate specimens with EDM notches using a $300 \mathrm{kHz}$ frequency shear horizontal angle beam sensor, and for a cylindrical shell specimen (4.2m inner diameter, $9 \mathrm{~mm}$ wall thickness) with discontinuities using a $40 \mathrm{kHz}$ frequency shear horizontal inter-digital sensor. The experimental reflection amplitudes were in good agreement with the analytical reflection amplitudes derived by the proposed model.
\end{abstract}

Key words: Ultrasonic Guided Wave, Reflection Coefficient, Plate

\section{Introduction}

The numbers of aged plants have been increasing in the power generation, oil, gas, chemical, and petro-chemical industries. A technique to efficiently evaluate integrity, especially for thickness, of many plate structures, for example pipes or tanks used in the plants, is becoming more important as present conventional non-destructive testing methods, i.e. radiographic testing, ultrasonic testing and eddy current testing, cannot meet these new inspection needs. This is because the methods naturally cover a narrow area, almost equal to their sensor size, and it is very time-consuming and cost-consuming to cover a long range or a wide area of the plate structures. Furthermore, if inspection points are placed in overhead locations, installation of scaffolds is needed before the inspection and then they must be removed afterwards; and except for radiographic testing, heat insulators must also be removed and then replaced for all inspection points.

One technique to overcome these issues is a long range inspection technique using an ultrasonic guided wave that was theoretically formulized by Gazis ${ }^{(1)(2)}$. In recent years, several approaches have been proposed and some techniques have been adopted commercially $^{(3)(4)(5)(6)}$. In these inspection techniques, a ring sensor is used, and the discontinuity size is measured as a cross-sectional area change of the pipe by using

${ }^{*}$ Received 27 Sep., 2013 (No.13-0077) [DOI: 10.1299/jmmp.7.601]

Copyright $@ 2013$ by JSME 
amplitude of a reflected guided wave. However, the amplitude of a reflected guided wave is known to depend on the length of the discontinuity; therefore the authors previously proposed a reflection model for the guided wave in a pipe ${ }^{(7)(8)}$. On the other hand, for tanks, the reflection amplitude also depends on the relative position between the sensor and the discontinuity.

Based on this background, the authors propose an analysis method to obtain the receiving gain of the guided wave. The model is based on combining the reflection from a discontinuity and a guided-wave field of a fundamental shear horizontal wave. In this paper, first, the details of the combined model are described and then the model is verified by some experiments.

\section{Modeling of the guided-wave field and reflection from a discontinuity}

\subsection{Modeling of reflection from a discontinuity}

In general, the reflection coefficient $R_{0}$ and transmission coefficient $T_{0}$ of an ultrasonic bulk wave from medium 1 to medium 2 are given by $R_{0}=\left(z_{02}-z_{01}\right) /\left(z_{02}+z_{01}\right)$ and $T_{0}=2 z_{02} /\left(z_{02}+z_{01}\right)$, respectively, where $z_{01}=\rho_{1} c_{1}$ is the specific acoustic impedance of medium 1 ( $\rho_{1}$ and $c_{1}$ are density and velocity of medium 1 ) and $z_{02}=\rho_{2} c_{2}$ is the specific acoustic impedance of medium $2\left(\rho_{2}\right.$ and $c_{2}$ are density and velocity of medium 2 ).

The reflection coefficient $R$ and transmission coefficient $T$ of a fundamental shear horizontal guided wave propagating in a medium of finite thickness are given by $R=\left(z_{2}-z_{1}\right) /\left(z_{2}+z_{1}\right)$ and $T=2 z_{2} /\left(z_{2}+z_{1}\right)$, respectively, where $z_{1}=\rho_{1} c_{1} A_{1}$ and $z_{2}=\rho_{2} c_{2} A_{2}$ are respective characteristic acoustic impedances of medium 1 and medium 2 , and $A_{1}$ and $A_{2}$ are respective cross-sectional areas of medium 1 and medium 2 as shown in Fig. 1(a) ${ }^{(9)}$. The reason why the reflection and transmission coefficients are represented by the simple equations is because the fundamental shear horizontal guided wave has same displacement through the medium and the propagation direction is parallel to the medium interface. Therefore, for guided waves, reflection occurs at a discontinuity even if the medium density or velocity is not changed between medium 1 and medium 2 , i.e. $\rho_{1}=\rho_{2}$ and $c_{1}=c_{2}$. If the discontinuity is a rectangular shape, the amplitude reflection coefficient is given by Eq.(1), like the total amplitude reflection coefficient of a bulk wave: ${ }^{(10)}$

$$
R=\left(\frac{z_{2}}{z_{1}}-\frac{z_{1}}{z_{2}}\right) / \sqrt{4 \cot ^{2} \alpha+\left(\frac{z_{2}}{z_{1}}+\frac{z_{1}}{z_{2}}\right)^{2}}
$$

where $\alpha=2 \pi a / \lambda, a$ is axial length of the discontinuity (medium 2) and $\lambda$ is the wavelength of the guided wave.

If the cross-sectional area of the discontinuity is slightly changed due to corrosion, for example, the reflection coefficient from the whole discontinuity is calculated by spatial integration of the waves reflected from the divided regions as shown in Fig. 1(b) and (c). Assuming that the guided wave is continuous and multiple reflection waves having over two reflection times can be ignored because of their low amplitude, the reflection wave from the whole region is given by Eq.(2):

$$
f(x, t)=\sum_{i=1}^{N}\left(\prod_{m=1}^{i-1}\left(\frac{2 z_{m+1}}{z_{m+1}+z_{m}} \frac{2 z_{m}}{z_{m+1}+z_{m}}\right) \frac{z_{i+1}-z_{i}}{z_{i+1}+z_{i}} \exp j\left(\omega t-k\left(x-2 x_{i-1}\right)\right)\right)
$$

where $x$ is axial position, $t$ is time, $z_{i}$ is characteristic acoustic impedance of the $i$ th small region, $\omega$ is angle frequency, $k$ is wave number and $j$ is imaginary unit.

Example analysis results of two types of typical discontinuities (rectangular and semi-elliptical) are shown in Fig. 2. For the simplest rectangular discontinuity, the reflection coefficient has its maximum value when the axial length of the discontinuity is $(2 n-1) / 4$ 
times the wavelength $(n=1,2 \cdots)$. On the other hand, the reflection coefficient has its minimum value at $n / 2$ times the wavelength $(n=1,2 \cdots)$. This periodicity is caused by interference between the anti-phase reflection wave from the position where the thickness is decreasing and the in-phase reflection wave from the position where the thickness is increasing. The reflection waves from the semi-elliptical discontinuity are also periodically changed for the same reason. But, its reflection coefficient decreases slightly for a long axial length. Therefore, the axial discontinuity length or shape is estimated by measuring the relationship between the frequency and reflection coefficient.

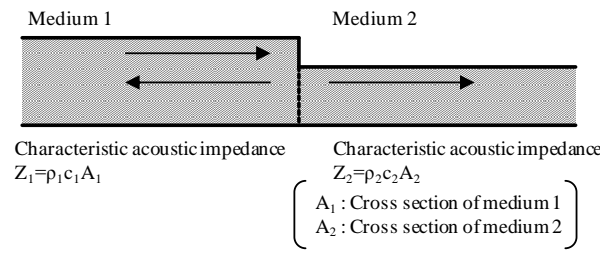

(a) Incidence, reflection and transmission of guided wave

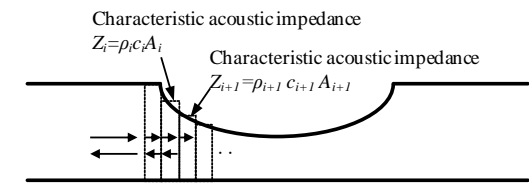

(b) 2D analysis model

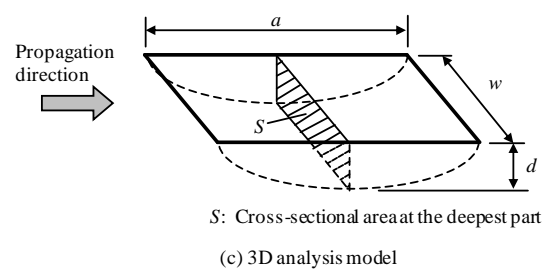

Fig. 1 Analysis models of guided-wave reflection
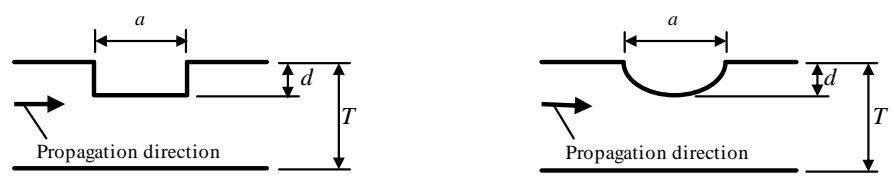

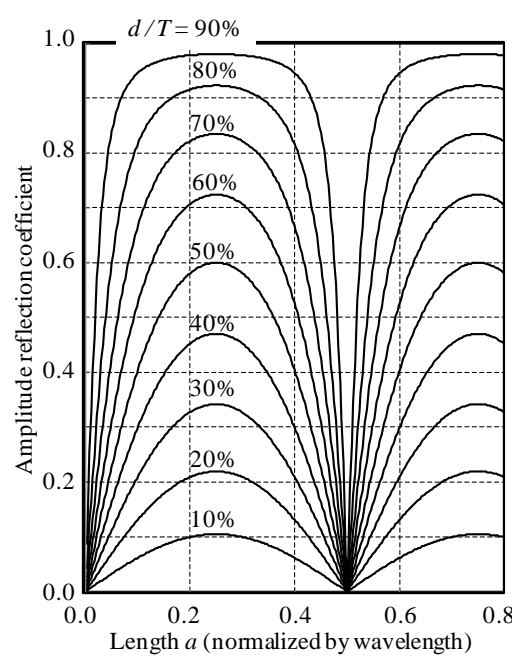

(a) Rectangulardiscontinuity

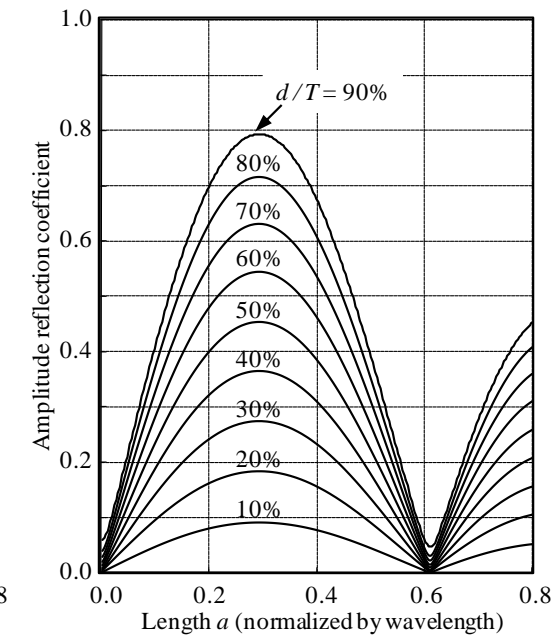

(b) Semi-elliptical discontinuity

Fig. 2 Reflection coefficients calculated by the proposed model 


\subsection{Modeling of the wave field}

For a bulk wave, the amplitude of the reflected wave changes in a complicated way if a discontinuity is located in the near-field region. Conventionally, the size of a discontinuity can be evaluated as equivalent-defect-diameter and the diameter of a circular defect shape is assumed to be a circle using the DGS (distance-gain-size) diagram ${ }^{(11)}$. This concept can be used for a guided wave also.

A guided wave has two characteristics. First, the amplitude of the guided wave is decreased in proportion to the square root of the distance from the source because the guided wave is a cylindrical wave. Second, the sound source is homogeneous through the wall and it can be treated as a line source on a two-dimensional plane as shown in Fig. 3. Therefore, the reflected wave received by the wave source (a transducer) is calculated by the Kirchhoff method using the following procedure. First, the vibration of element $q$ on the discontinuity is expressed by Eq.(3)

$$
y_{q}(t)=\sum_{p} \frac{A_{p}}{\sqrt{r}} \exp j(\omega t-k r)
$$

where $r=\left|r_{1}-r_{0}\right|, \quad r_{0}$ is the position vector of element $p$ on the wave source, $r_{1}$ is the position vector of element $q$ on the reflection source, $\omega$ is angular frequency, $t$ is time, $j$ is imaginary unit, and $k$ is wave number. Next, the vibration $x_{p}(t)$ of element $p$ on the wave source (transducer) is expressed by Eq.(4)

$$
x_{p}(t)=\sum_{q} \frac{1}{\sqrt{r^{\prime}}} y_{q}(t) \exp \left(-j k r^{\prime}\right)
$$

where $r^{\prime}=\left|r_{2}-r_{1}\right|$, and $r_{2}$ is the position vector of element $p$. Finally, the amplitude $C$ of the reflected wave received by the wave source is expressed by Eq.(5).

$$
C=\sqrt{\sum_{p} x_{p}^{2}}
$$

When the reflection source is in front of the wave source, the DGS diagram of the guided wave that has $k a=4.49$ ( $k$ is wave number and $a$ is the half length of the sound source) is shown in Fig. 4(a). In the figure, the horizontal axis is normalized distance (distance $L$ is normalized by wavelength $\lambda$ ), and the vertical axis is relative amplitude normalized by the amplitude of the reflected wave from the reflection source $w / W=\infty$ ( $w$ is width of the reflection source and $W$ is width of the wave source) at the normalized distance $=1$. The amplitude from the reflection source $w / W=\infty$ decreases $10 \mathrm{~dB}$ for each power of ten change in distance, but the amplitude from the finite width of the reflection source decreases $20 \mathrm{~dB}$ for each power of ten change in distance at the far-field, and the relative amplitude is proportional to the width of the reflection source. The relationship between the normalized lateral distance $D / \lambda$ and relative amplitude at $L / \lambda=100$ is shown in Fig. 4(b).

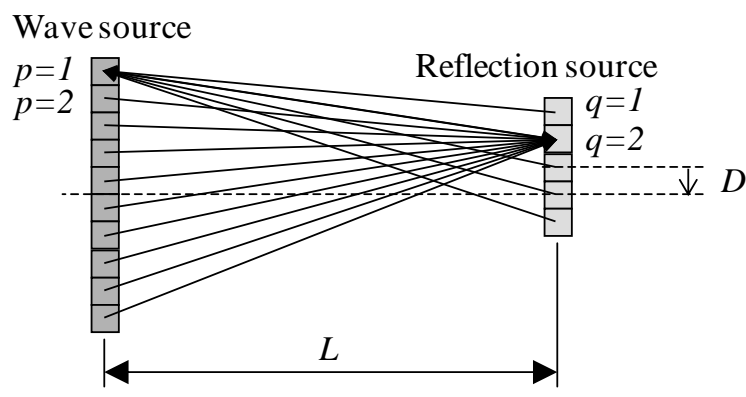

Fig. 3 Analysis model of guided-wave field 


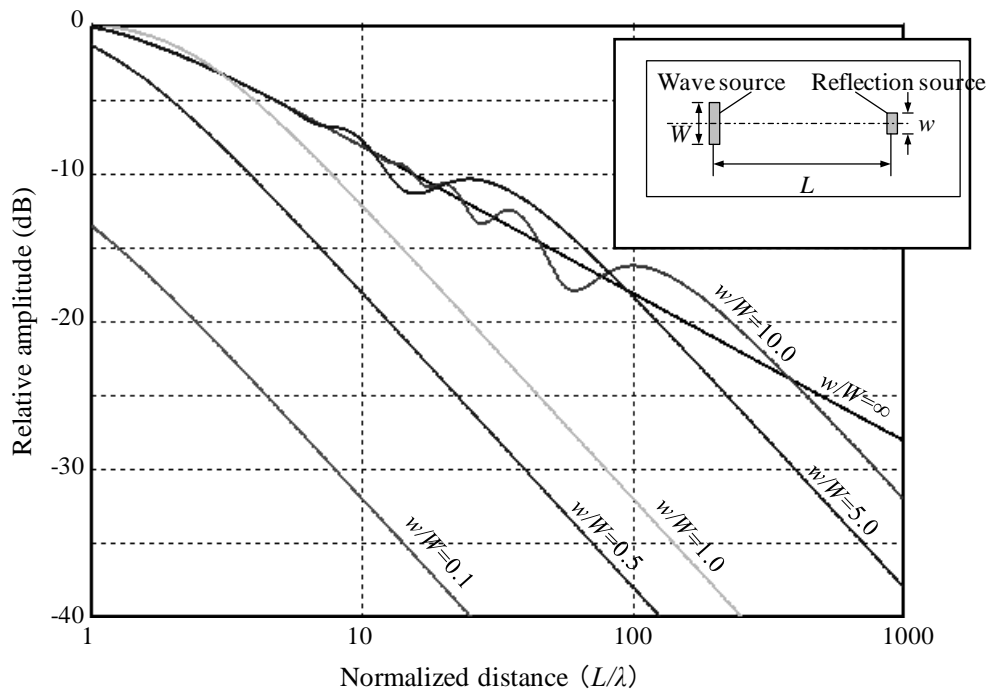

(a) Distance-gain-size diagram: $k a=4.49$ where $k$ means wave number and $a$ means half length of wave source

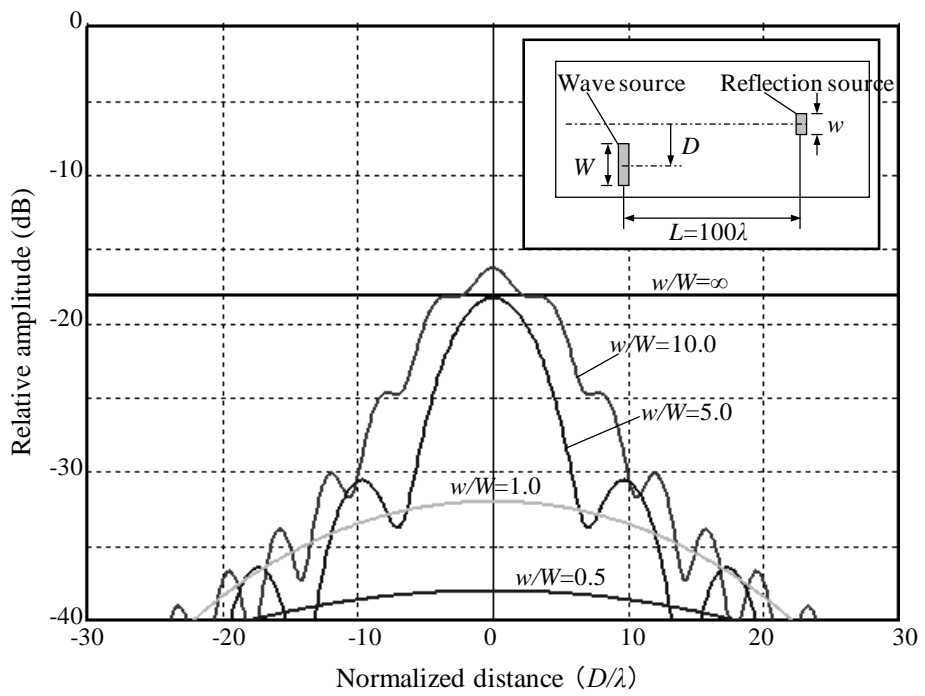

(b) Relationship between lateral distance and amplitude: $k a=4.49, L / \lambda=100$.

Fig. 4 Analysis results of relative amplitude from several size discontinuities by the wave field model

\section{Experiments}

\subsection{Experiment with plate specimens}

In order to measure amplitude of the reflected wave from rectangular discontinuities, 2 types of stainless steel plate specimens (thickness $=4 \mathrm{~mm}$ ) with artificial discontinuities were fabricated. One type specimen had 4 discontinuities with width of $100 \mathrm{~mm}$ and height of $h=0.5 \mathrm{~mm}$ to $h=2.0 \mathrm{~mm}$ in steps of $0.5 \mathrm{~mm}$ (Fig. 5(b)). The other type specimen had 2 discontinuities with width of $100 \mathrm{~mm}$ and each height of $h=2.5 \mathrm{~mm}$ or $h=3.0 \mathrm{~mm}$.

A block diagram of the experimental system is shown in Fig. 5(a). An arbitrary function generator, power amplifier, diplexer receiver amplifier and A/D converter (maximum sampling rate $=1 \mathrm{GS} / \mathrm{s}$, vertical resolution $=8$ bits) configured the system. The transducer was a shear-vibration type with a resonance frequency of $300 \mathrm{kHz}$. It was bonded on an acrylic wedge with a refraction angle of 90deg which matched the shear horizontal wave velocity.

The transducer was placed on a plate specimen using viscosity medium coupling, opposite the fabricated discontinuities, and a normal load was given until the amplitude 
became stable. Then, the transducer was placed 700mm from the discontinuities, and the reflected wave was recorded. The amplitude of the reflected wave from the plate end was also measured. In the experiments, the transducer was driven by a $300 \mathrm{kHz}$ tone-burst wave.
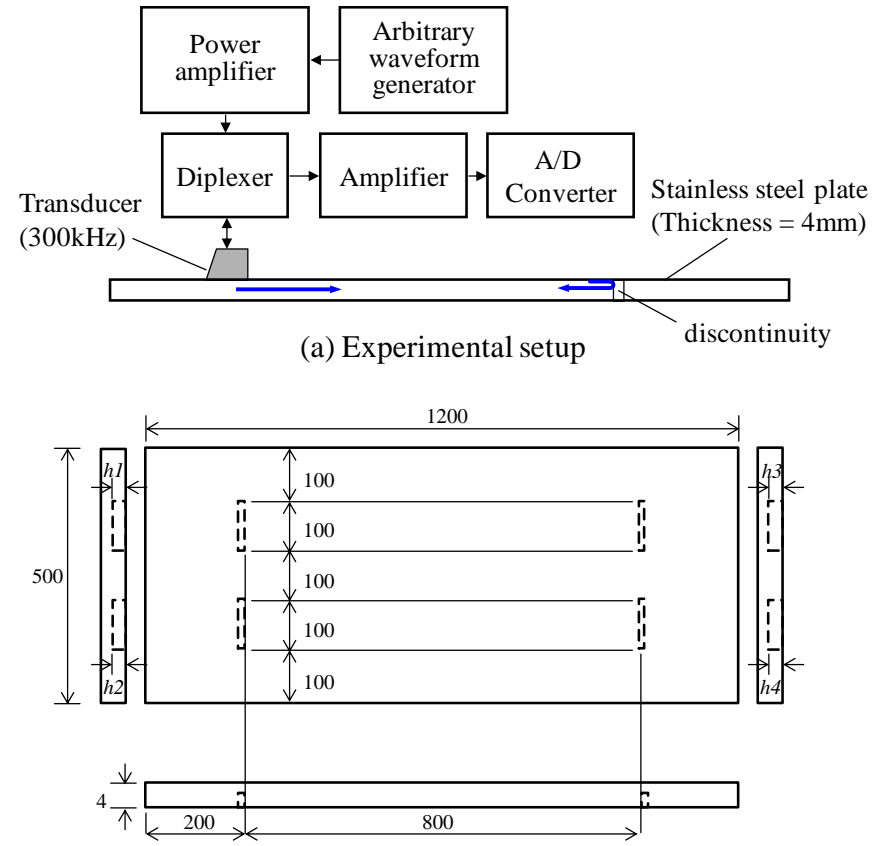

(b) Shape of specimens

Fig. 5 Experimental setup and shape of plate specimens (unit: $\mathrm{mm}$ )

The amplitude reflection coefficients of rectangular discontinuities obtained from the experiment with the plate specimens are shown in Fig. 6. The filled circles are experimental results normalized by the reflection amplitude of the through-thickness discontinuity with width of $100 \mathrm{~mm}$. Solid lines are theoretical results obtained by Eq.(1). In this experiment, the frequency of the guided wave was $300 \mathrm{kHz}$ and the length of the rectangular discontinuity was $1 \mathrm{~mm}$, then the length of the discontinuity normalized by the wavelength ( 10.5mm) was about 0.1 . In this figure, experimental results are plotted between analytical values of $1.0 \mathrm{~mm}$ length discontinuity and $1.5 \mathrm{~mm}$ length discontinuity. Nominal length of the discontinuity was $1 \mathrm{~mm}$, and the results obtained for the proposed model were approximately in agreement with the experimental results. 


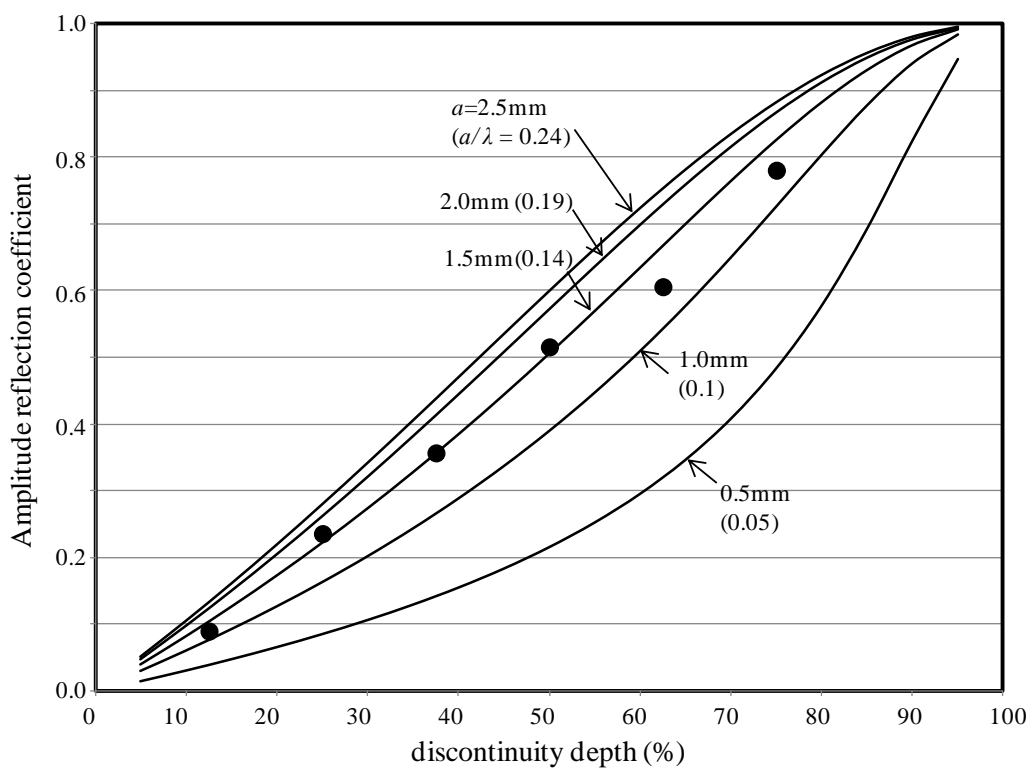

Fig. 6 Amplitude reflection coefficients of rectangular discontinuities on plate specimens (frequency = $300 \mathrm{kHz}$ ): experimental values are normalized by signal amplitude of through-thickness discontinuity

\subsection{Experiment with the cylindrical shell specimen}

The guided-wave system and sensor are shown in Fig. 7(a). The system included arbitrary function generators, power amplifiers, an A/D converter and a computer for control. The sensor had 8 inter-digital transducer groups $(4 \times 2$ rows). The inter-digital transducers had a shear mode element and the fundamental shear horizontal mode guided wave with no dispersion was excited.

In order to control the transmission direction, 4 pairs of transducers were set on the cylindrical shell specimen with a separation of $20 \mathrm{~mm}$ (Fig. 7(b)), which is about one-quarter of the wavelength of the $40 \mathrm{kHz}$ shear horizontal mode, and a one-quarter period delayed waveform was applied to each pair. Backward transmission was actively suppressed by the inter-digital transducers according to the following principle. A synthetic traveling wave in the plus (right hand) direction $A^{+}(x, t)$ and a synthetic traveling wave in the minus (left hand) direction $A^{-}(x, t)$ are given by Eqs.(6) and (7), respectively:

$$
\begin{aligned}
& A^{+}(x, t)=A_{1} e^{j(\omega t-k x)}+A_{2} e^{j\left(\omega\left(t-\frac{T}{4}\right)-k\left(x-\frac{\lambda}{4}\right)\right)} \\
& A^{-}(x, t)=A_{1} e^{j(\omega t+k x)}+A_{2} e^{j\left(\omega\left(t-\frac{T}{4}\right)+k\left(x-\frac{\lambda}{4}\right)\right)}
\end{aligned}
$$

where $x$ is the axial coordinate of the pipe, $A_{1}$ and $A_{2}$ are amplitudes of the transmitting wave of positions 1 and 2, respectively, $\omega$ is angle frequency, $t$ is time, $k$ is wave number, $T(=2 \pi / \omega)$ is period, $\lambda(=2 \pi / k)$ is wavelength, and $j$ is imaginary unit. For $A_{1}=A_{2}$ in Eqs.(6) and (7), a synthetic wave traveling in the plus direction is doubly super-positioned, and a synthetic wave traveling in the minus direction is suppressed to zero. This is shown in Fig. 8.

In practice, the transmitting signals used for inspection are not continuous waves but burst waves that have a limited duration. Then, the waveform applied to the front row of the transducer pairs was the inverse of the waveform applied to the rear row and it had a one-quarter period delay. In this method, the amplitude of the synthetic traveling wave in the plus direction could not be completely doubled, but the amplitude of the synthetic traveling wave in the minus direction could be completely decreased to zero in theory. For inspection, distinguishing between the reflection wave from one direction (signal) and the 
reflection wave from another direction (noise or false signal) is important. Therefore, almost the same processing was performed for received signals.
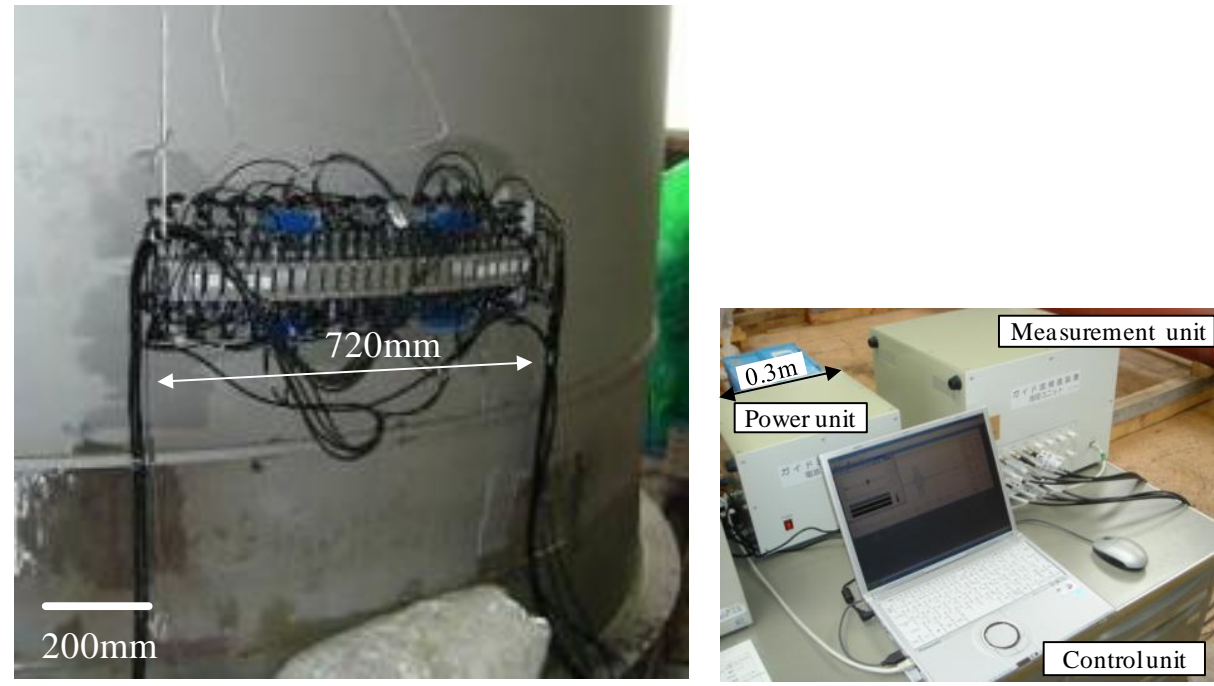

(a) Guided-wave system and sensor on a cylindrical shell specimen

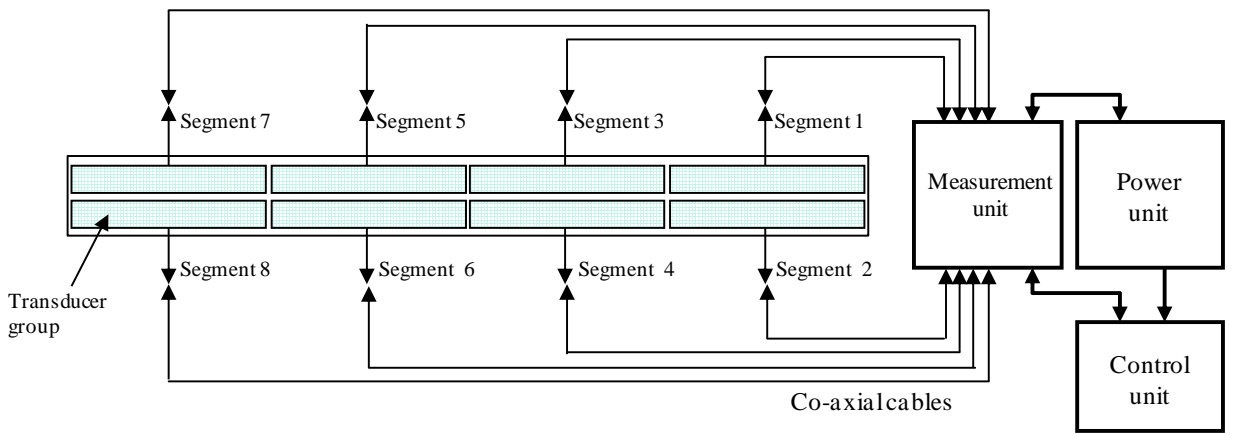

(b) System configuration

Fig. 7 Photo of the guided-wave system and sensor and a block diagram of the system
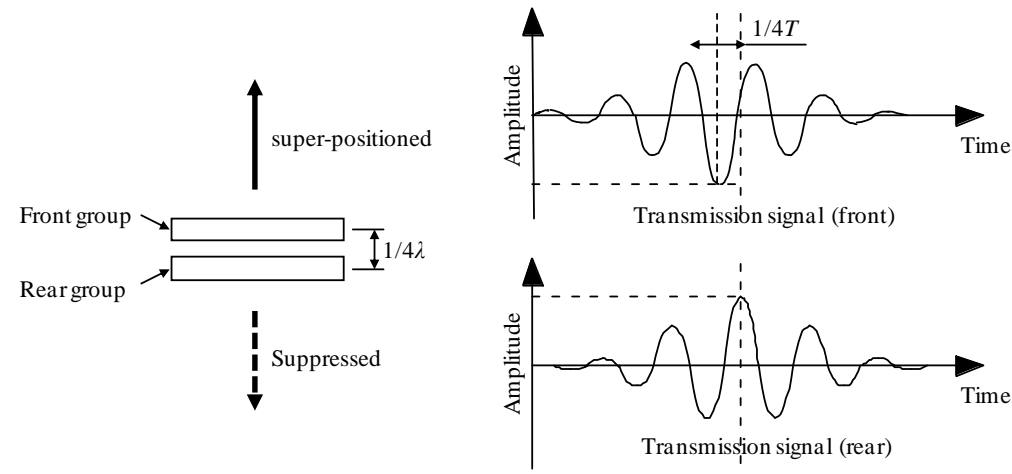

Fig. 8 Transmission direction control by the inter-digital transducers

A cylindrical shell specimen was used as shown in Fig. 9. The specimen was SUS304, with $4200 \mathrm{~mm}$ inner diameter, $2800 \mathrm{~mm}$ height, and $9 \mathrm{~mm}$ nominal wall thickness. Two axial butt welds were positioned, one at $0 \mathrm{deg}$ and the other at 180deg. Three different shapes of artificial discontinuities ( $\mathrm{O}, \mathrm{A}, \mathrm{R})$ were fabricated on the inner diameter (ID) side of the specimen. The guided-wave sensor was positioned at 6 locations (P1 to P6), and the guided 
wave was transmitted in the direction indicated by the arrows. The reflection signal amplitude from the discontinuity was measured at several frequencies.

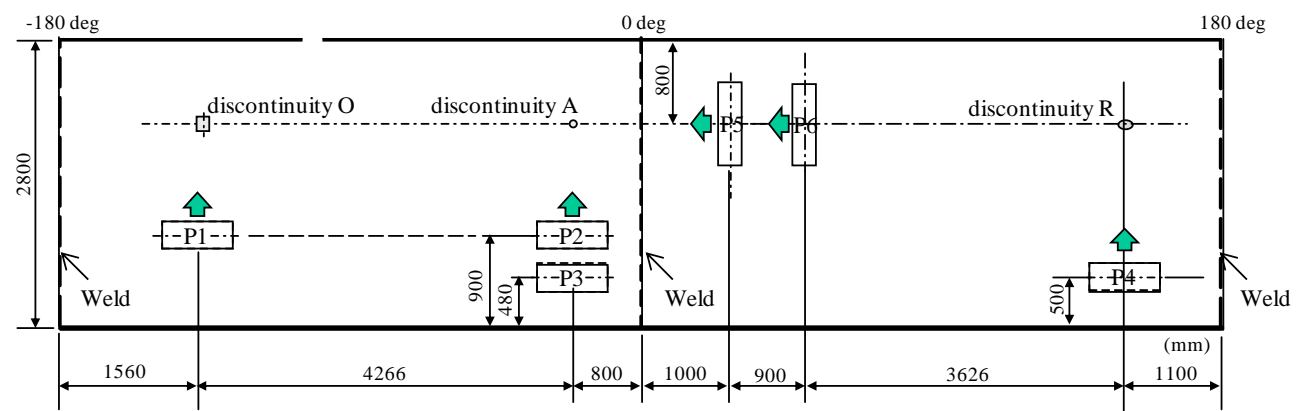

(a) Positions of discontinuities and positions of the sensor (P1 to P6) on cylindrical shell specimen

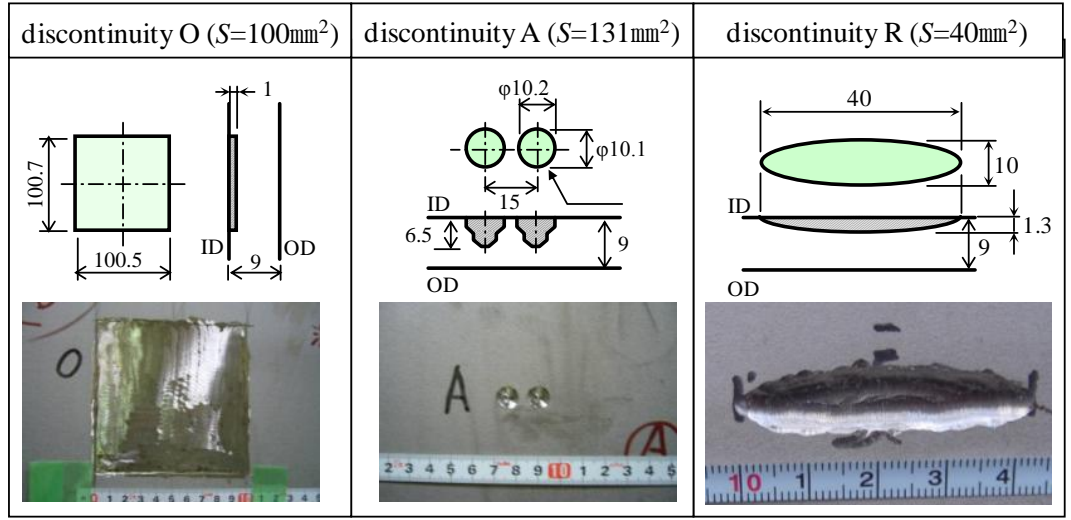

(b) Discontinuity shapes

Fig. 9 Cylindrical shell specimen and artificial discontinuities

The relative reflection amplitudes of the discontinuities obtained from the experiment with the cylindrical shell specimen are shown in Fig. 10. Filled marks are experimental results. Solid lines are analytical amplitudes obtained by Eq.(1) or Eq.(2), and Eq.(5). For comparison of the experimental amplitudes and the analytical amplitudes, the experimental amplitudes were corrected by using the ratio between the analytical amplitude of $w / W=\infty$ and an experimental amplitude of the top end of the cylindrical shell.

In the reflection coefficient analysis, sizes of discontinuities were precisely measured by the replica method, and were modeled by the values of Table 1. For example, discontinuity $\mathrm{O}, 100 \mathrm{~mm}$ square length and $1 \mathrm{~mm}$ depth, was approximately modeled as a $1 \mathrm{~mm}$-step discontinuity, because reflection waves at the decreasing step and increasing step hardly interfere with each other since the difference of propagation distance was $200 \mathrm{~mm}$ and the duration time of waveform was about 3 cycles as shown in Fig. 8. Discontinuities A and $\mathrm{R}$ were modeled as a semi-elliptical shape, with depths of $6.5 \mathrm{~mm}$ and $1.27 \mathrm{~mm}$, respectively, and width of $10 \mathrm{~mm}$. Axial weld, i.e. excess weld metal, was also modeled as a semi-elliptical shape, with length of $14 \mathrm{~mm}$ and depth of $-0.8 \mathrm{~mm}$, where the minus value means thickness was increasing.

Widths of the discontinuities for the wave field analysis were modeled by the values of Table 1 . The width $w$ of discontinuity A, a pair of $10 \mathrm{~mm}$-diameter drilled holes, was modeled as $20 \mathrm{~mm}$. Width of the axial weld was defined as $\infty$. The guided-wave sensor was modeled as $W=720 \mathrm{~mm}$ width, $40 \mathrm{kHz}$ frequency. The solid lines in Fig. 10 were calculated by this wave field analysis, and the values were corrected by the reflection coefficients in Table 1.

In Fig. 10, the relative amplitudes of discontinuities were in good agreement with 
analytical values, and the proposed model was verified. By using the model, detectable discontinuities were estimated by providing their shape and size, and in the future, it is expected that the shape and size can be estimated using the amplitude of received signals with multiple frequency transmissions.

Table 1 Reflection coefficients of discontinuities

\begin{tabular}{|c|c|c|c|c|c|}
\hline \multirow{3}{*}{ Discontinuity } & \multicolumn{4}{|c|}{ Reflection model } & Wave field model \\
\hline & \multirow{2}{*}{ Model } & Length (mm) & Depth (mm) & \multirow{2}{*}{$\begin{array}{l}\text { Reflection coef. } \\
\text { @40kHz (dB) }\end{array}$} & Width (mm) \\
\hline & & $a$ & $d$ & & $w$ \\
\hline Discontinuity O & Step & - & 1.0 & -24.6 & 100 \\
\hline Discontinuity A & \multirow{3}{*}{$\begin{array}{l}\text { Semi- } \\
\text { ellipse }\end{array}$} & 10 & 6.5 & -8.1 & 20 \\
\hline Discontinuity R & & 10 & 1.3 & -21.3 & 40 \\
\hline Axial weld & & 14 & -0.8 & -24.0 & $\infty$ \\
\hline
\end{tabular}

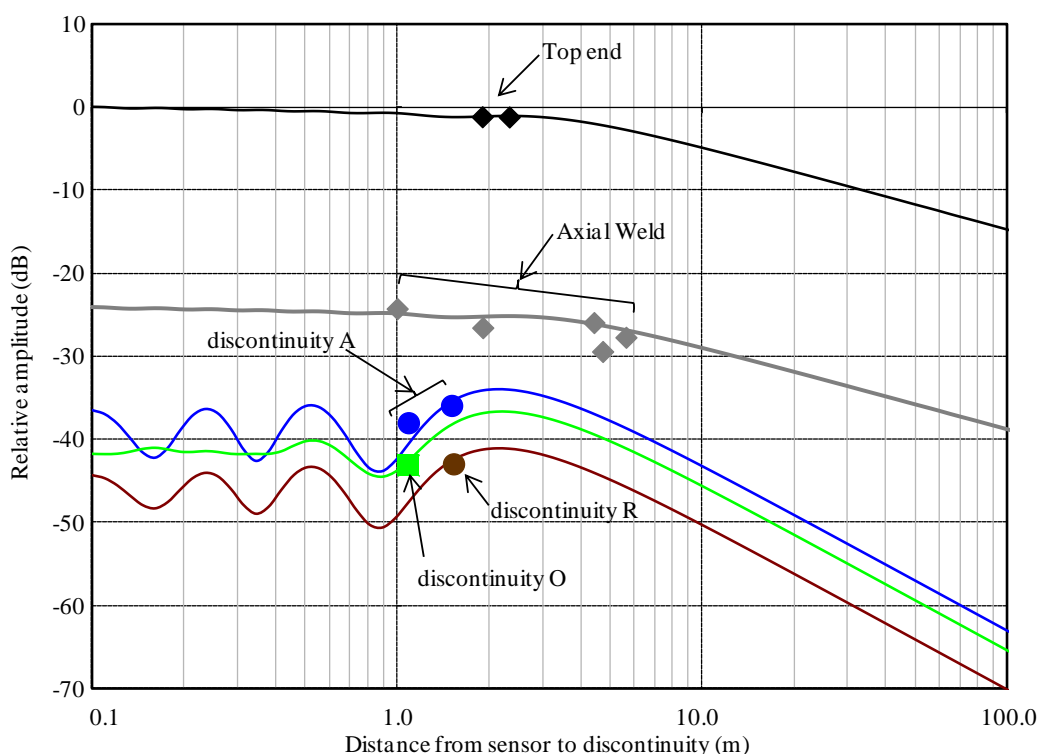

Fig. 10 Relative signal amplitude of discontinuities of the cylindrical shell specimen (frequency $=40 \mathrm{kHz}$ ): experimental results and analytical results obtained by the proposed model

\section{Conclusions}

A combined model for reflection from a discontinuity and for the guided-wave field of the fundamental shear horizontal guided wave in a plate structure was proposed. Some experiments and analyses were performed for $4 \mathrm{~mm}$-thick stainless steel plate specimens and for a cylindrical shell specimen with an inner diameter of $4.2 \mathrm{~m}$ and wall thickness of $9 \mathrm{~mm}$.

It was found that reflection coefficient of the guided wave from a rectangular discontinuity could be modeled in the same way as the total reflection coefficient of a bulk wave. For a discontinuity having a cross-sectional area that slightly changed, the reflection coefficient from the whole discontinuity could be calculated by spatial integration of the waves reflected from the divided regions. The experimental results were in good agreement with the analytical values obtained using the proposed combined model. 


\section{References}

(1) Gazis, D.C., Three-Dimensional Investigation of the Propagation of Waves in Hollow Circular Cylinders. I. Analytical Foundation, Journal of Acoustical Society of America, Vol.31, No.5 (1959), pp.568-573.

(2) Gazis, D.C, Three-Dimensional Investigation of the Propagation of Waves in Hollow Circular Cylinders. II. Numerical Results, Journal of Acoustical Society of America, Vol.31, No.5 (1959), pp.573-578.

(3) Mudge, P. J., Field Application of the Teletest Long-range Ultrasonic Testing Technique, Insight, Vol.43, No.2 (2001), pp.74-77.

(4) Sheard, M. and McNulty, A., Field Experience of Using Long-range Ultrasonic Testing, Insight, Vol.43, No.2 (2001), pp.79-83.

(5) Li, J. and Rose J. L., Angular-Profile Tuning of Guided Waves in Hollow Cylinders Using a Circumferential Phased Array, IEEE Transactions on Ultrasonic, Ferroelectrics and Frequency Control, Vol.49, No.12 (2002), pp.1720-1729.

(6) Rose, J. L., Sun, Z., Mudge, P. J. and Avioli, M. J., Guided Wave Flexural Mode Tuning and Focusing for Pipe Testing, Materials Evaluation, Vol.61, No.2 (2003), pp.162-167.

(7) Nagashima, Y., Endou, M. and Kouga, I., Discontinuity-length Sizing Potential Using Ultrasonic Guided Waves in Pipes, Proceedings of 15th International Conference on Nuclear Engineering, (2007), ICONE15-10637

(8) Nagashima, Y., Endou, M., Miki, M., Odakura, M. and Maniwa, K., Defect Sizing Method Using Ultrasonic Guided Waves in Pipes, Review of Progress in Quantitative Nondestructive Evaluation, Vol.28 (2008), pp.1583-1590

(9) Tomikawa, Y., Ultrasonic electronics vibration theory (in Japanese), (1998) pp.5-6, Asakura Publishing, Co., Ltd.

(10) American Society for Nondestructive testing, Nondestructive testing handbook third edition, volume 7, Ultrasonic testing, (2007), p.372

(11) Kimura, K. and Matsumoto, S., DGS Diagrams for Angle Probes, 3rd European Conference on NDT Florence, Vol. 3, (1984), pp. 93-102 